Hansell in the Annals of Ophthalmology of 1912, reports a case coming on during the course of acute inflammatory rheumatism. The patient died at the end of the third day. Cultures from the blood showed streptococci, but none could be found in the eyeball.

Graefe and Saemisch make the statement that it is not always possible to find a localized source of pus; and in such cases, one must assume that the bacteria have entered the body thru a mucous membrane without having excited evident inflammation.

Axenfeld tells us that metastatic ophthalmia is due primarily to the introduction of septic masses into the capillary vessels of the eye. In the binocular form, the first tissue to be infected is the retina; in the monocular, the uvea.

\title{
SOCIETY PROCEEDINGS
}

Reports for this department should be sent at the earliest date practicable to 1 r. Harry S. Gradle, 22 E. Washington St., Chicago, Illinois. These reports should present briefly the important scientific papers and discussions.

\section{COLLEGE OF PHYSICIANS OF PHILADELPHIA, SECTION ON OPHTHALMOLOGY.}

$$
\text { May 17, } 1921 .
$$

Dr. G. Oram Ring, Chatrman.

Double Coloboma (Nasal and Temporal) of the Optic Nerve.

Dr. B. Alex. Randall reported the history of a young woman, whose left eye, hyperopic with practically normal vision, has shown no change in the 12 years she has been under observation. Instead of a vertically oval disc as on the right, she presents a large horizontally oval disc with deep central porus and absorbing crescents of the choroid at both lateral margins. Within these, defined by no "scleroid ring," each disc margin shows a dark "bottomless" depression, sharp-cut outward: the one to the nasal side having the upper nasal vessels passing under its margin, somewhat as the other upper vessels are related to the steep edge of the porus. There is no trace of a colobomatous gap in the choroid or iris. Vision and refraction are equal in the two eyes.

It is a matter of interest that the grandmother presented to the nasal side of her left eye a horizontally oval lesion of the choroid, that might readily have been mistaken for coloboma. He had seen this, however, in its first stage as a patch of choroiditis, in the center of which a bleb of retina was detached by exudate which ruptured into the vitreous and was absorbed, the vague yellow patch of inflamed choroid then going on to atrophy, with pigment heaping at its margins. The first stage of this condition is beautifully shown in Jaeger's plate, but so far as he knew there is no recorded observation of the later stage, as he had the chance to observe it thru a series of years.

\section{Capsulo-Muscular Advancement With-} out Incision.

Dr. S. Lewis ZIEgLer exhibited a sccond case of his simplified operation to supplement the one shown before the Section about a year ago.

A capsulo-muscular advancement of the external rectus of left eye was performed by suture alone, without incision, which brought the eye almost into position. This was accomplished by grasping the muscle thru the conjunctiva, about $12 \mathrm{~mm}$. back from the cornea, and entering a double armed suture by a whip-stitch, first thru one margin of the muscle and then thru the other. The needles were then carried forward over the conjunctiva in a line parallel with each muscle margin and inserted in the sclera at the junction, deeply enough to secure a firm scleral anchorage. The suture was tied in a double surgical loop, and the tissues drawn forcibly forward until the necessary reposition was secured, when the second loop of the knot was tied. 
This advancement was supplemented by an exploratory incision over the internal rectus of the same eye thru which the tenotomy hook was passed and the muscle stretched above and below. In doing this a small adhesion of the capsule above was found and incised, which relieved the hypertropia and brought the eye back to normal level. A partial tenotomy of this muscle was also performed which increased the freedom of excursion.

A Liebreich patch was worn for a few days. A slight crumpling of the muscle was evident for one week. There was no inflammatory reaction but redness persisted for one month. The single external suture was easily removed on the twelfth day.

Examination now reveals excellent parallel movement of the eyes in all directions with complete restoration of excursion to the left side. The eyes are perfectly straight, altho a slight tendency to overconverge is sometimes noticed when glasses are omitted.

The result shows that three difficulties have been overcome:

(1) Overconvergence from $s u p$ pressed image, O. S., relieved by capsulo-muscular advancement of external rectus.

(2) Old contraction of capsule and internal rectus from disuse, relieved by stretching and partial tenotomy.

(3) Hypertropia from adhesion of capsule at upper margin of internal rectus $O$. S., relieved by division of the adhesion.

Discussion. Dr. H. F. Hansell. The simplicity of Dr. Ziegler's operation makes a strong appeal for its performance in preference to those in which the conjunctiva is incised, the capsule of Tennon opened, and the tendon separated at its attachment, or muscular tissue excised. In this patient and in the one that Dr. Ziegler previously exhibited to the Section, the advancement or shortening was combined with tenotomy of the internus. I suggest that equally good or better traction on the sutures could be obtained with the scleral anchorage made by almost hor- izontally inserted needles rather than by their vertical insertion.

Dr. Zentmayer said that, in this case, as well as the one previously shown by Dr. Ziegler, the capsulo-muscular advancement had been combined with a tenotomy of the antagonist. Almost any advancement operation, if combined with a tenotomy of the antagonist will give a good primary result. Until a case is shown in which no tenotomy has been done, it will be impossible to judge of the merits of Dr. Ziegler's ingenious procedure.

Dr. Ziegler said, in reply to Dr. Hansell, that he purposely did not converge the sutures before anchoring, as this would distort the tissues at the point of fixation and would not maintain the parallel traction which he considered an essential factor in securing a good result. In answer to Dr. Zentmayer's query as to how much of the effect is due to advancement of the muscle and how much is due to division or weakening of the opposing muscle, it is only necessary to measure the effect of the advancement as soon as the operation is completed, providing binocular vision is good enough to elicit diplopia.

Ile wished to emphasize the need of searching for capsular adhesions on the side of the contracted muscle which often nullify all efforts to correct the strabismus. This can be done by passing the tenotomy hook thru a small incision in the conjunctiva and exploring the tissues both above and below the muscle. If the hook fails to pass freely, the scissors can be slipped into the conjunctival opening and the adhesion gently divided without disturbing the relations. It is often surprising how this simple maneuvre may convert an apparent failure ino a success. In this case it relieved the hypertropia chiefly.

\section{Electrically Tinted Optical Glass.}

Dr. Sidney L. Olsho (by invitation) presented specimens of optical lenses treated by exposure to X-rays produced by a special, selfrectifying, air and water cooled tube, in an apparatus recently perfected. Under this process, American crown glass assumes a light 
amber tint after an exposure to 100 , $50 \mathrm{~K}$. V. for two minutes. Longer exposure deepens the color. The process is therefore under control. German optical glass develops an amethyst cast. For details of this process see Dr. Olsho's paper, p. 644, A. J. O., September, 1921 .

Discussion. Dr. Zentmayer. It seems this glass is an artificially produced amethyst glass offered as a substitute for amethyst glass produced by the action of the sun's rays on certain kinds of window glass. My recollection is that in the table put out by the Bureau of Standards, amethyst glass ranks low in its power of absorption of ultraviolet rays, whereas for this glass the claim is that it absorbs a very large percentage of these rays.

Dr. OIsho stated that the chart submitted shows the affinity of these lenses for the absorption of ultraviolet rays. The amber tinted lenses are the more effective.

\section{Multifocal Lenses.}

DR. Sidney L. Olsho (by invitation) presented specimens of finished multifocal lenses, which are designed to take the place of invisible bifocal lenses and were first described to the Franklin Institute, in this city, by their inventor, $\mathrm{H}$. O. Gowlland, of Montreal. The lower or reading portion of this lens presents a continuous variable curve. The periphery of the lower portion is indiscernible. From this periphery there is a gradual accretion of power to the reading centre, the limit being the addition ordered. The lenses are toric in form, ground on a constant -6 D. base, with additions up to $3 \mathrm{D}$. Any combination is secured by surfacing the other side.

The advantages claimed for this lens are: The possibility of embodying in one lens, powers for the intermediate distances between infinity and the reading distance. There is no sharp jump from the distance to the near focus. The wearer is supposedly able to select a focus for any intermediate distance at will, or may bring matter requiring closest scrutiny into the zone controlled by the carefully positioned centre. The field for close work is more restricted than in the ordinary bifocal, but this restriction is to some extent counterbalanced by the multiple range. These lenses are known under the trade name "Ultifo."

\section{Groenouw's Nodular Keratitis.}

Dr. Wm. Zentmayer presented a woman, aged sixty-eight years, who had discovered accidentally four years ago that the vision in the left eye was much impaired. There was nothing in the personal or family history bearing on the ocular condition. The cornea of the eye showed a group of capacities of greenish-gray color, varying in size from one-half to three $\mathrm{mm}$. and of different contours, one or more of them having a sigmoid shape. The lesions were situated immediately beneath the epithelium, which was elevated irregularly, giving to the surface of the cornea an unevenness. There was no ciliary injection and no history of past inflammation. The right eye showed the beginning of a similar condition. He expressed the belief that the process was probably a dystrophy, but doubted whether tuberculosis was an etiologic factor in this particular type of degeneration.

\section{Central Exudative Retinitis.}

Dr. Wm. Zentmayer exhibited a man, aged fifty-one years. Dimness of vision of the left eye was first noticed January 1, 1921. In November, 1920, he underwent an ocular examination in the railroad service, and the vision at that time was normal. The loss of vision was accompanied by marked metamorphopsia and halos. One year ago the patient was ill for several weeks, the condition being diagnosed as nervous prostration. Aside from the ordinary diseases of childhood, this was his only illness. No history of injury. Family history negative, von Pirquet and Wassermann negative. V. R. E., 6/5; L. E., 6/30. External conditions normal. Field of vision, right eye slight temporal contraction, left eye temporal contraction with a central positive scotoma.

Ophthalmoscope: The right eye showed in the macular region a group 
of grayish-white, irregular shaped lesions averaging about the diameter of the first branch of the vessels, occupying an area two-thirds the diameter of the disc and having somewhat of a rosette arrangement. In the left eye there was an exudation in the macula about four times the disc in size, with an elevation of about $1 \mathrm{~mm}$. There was a corona of hemorrhage surrounding its margin. Because of the normal functioning of the right eye, the lesion was looked upon as a hyalin degeneration. In the left cye the etiologic factor had not as yet been determined.

\section{Foreign Body Occupying the Orbit and Accessory Sinuses.}

DR. T. B. Holloway gave the history of an injury to the right eye in a boy, aged sixteen years, by the explosion of a piece of wrought iron pipe, which had been loaded with powder. At the time of the explosion he was ten feet from the improvised cannon. Fxamination July 26, 1920, three weeks after the injury, showed a scar, $5 \mathrm{~cm}$. in length, which extended across the middle of the nose. Moderate proptosis of the right eye and marked chemosis of the bulbar conjunctiva. Cornea hazy, anterior chamber deep, iris discolored, and pupil vertically oval and inactive. Complete loss of outward rotation and distinct impairment of the other ocular muscles. A grayish refex from the vitreous by oblique illumination. Tension minus 2 ; no 1 . p. No mass could be felt about the globe, there was slight tenderness over the lower and inner part of the orbit. Left eye negative, vision $6 / 6$.

An X-ray examination revealed an enormous foreign body, the lower end of which appeared to be free in the nasal cavities with the upper end lodged in the orbit. As extraction from the orbit would be impossible without breaking down the inner wall, Dr. George Fetterolf was called in consultation. He reported "at nasal examination there could be seen and felt a metallic mass at the anterior part of the right nasal cavity between the middle meatus and the septum. Under ether a pair of strong forceps was applied to the mass and by the use of considerable force and after loosening by lateral movements, withdrawal of the foreign body was effected. It proved to be a horizontal section of an iron pipe measuring $42 \mathrm{~mm}$. in length by $12 \mathrm{~mm}$. in breadth. Removal was followed by a small amount of bleeding."

When the patient was last seen on February 17, 1921, the globe still showed a slightly pinkish-yellow injection and distinct evidence of atrophy. No view of the fundus was possible.

\section{An Illuminated Perimeter with Campi- meter Features.}

Drs. C. E. Ferree and G. Rand, of Bryn Mawr College (by invitation), exhibited an apparatus devised in response to a request from a committee of the American Ophthalmological Society, for a feasible means of illuminating the perimeter arm with light of good intensity and quality, so that every point on the arm in any meridian in which it might be placed would receive equal intensities of light. Intensity and quality of illumination, however, are only two of the factors which influence the results of the perimeter determination. In devising the apparatus provisions have bcen made, therefore, for the control of other factors which are of importance. Some of these controls are:

(1) Fvery point of the perimeter arm in any meridian in which it may be placed receives light of equal intensity and of approximately daylight quality by ammeter and rheostat control. The instrument can be operated on any 110 volt circuit and the results obtained with it are absolutely independent of the variability of daylight illumination.

(2) Provision is made so that the eye, just before receiving the color stimulation, is preexposed to a surface of the brightness of the color as seen at the limits of the color field. The colored stimulus is surrounded also by a field of this same brightness. Variability in these two factors alone may change the limits as much as 20 
degrees in some meridians. A further advantage is gained by making the background of the same brightness as the color:-the stimulus disappears completely when the limits of sensitivity to the color is reached, instead of turning into a gray, concerning the colorlessness of which the patient is apt to be in doubt. This gives the effect of the disappearance type of photometer and adds greatly to the ease and certainty of making the judgment.

(3) Accurate provision is made for maintaining the eye at the center of the sphere in the surface of which lies the perimeter arm and of holding a constant and steady fixation. The quick adjustment of the cye is facilitated by three rack and pinion motions. Constancy of relation of the meridians of the retina with the meridians of the field of vision as laid off by the perimeter arm is secured by an especially devised, shape-conforming headrest.

(4) An attachment is provided for controlling the fixation of patients with a central scotoma or pathologic blind area.. With the eye properly adjusted for taking the fields, these patients are not able to see a central fixation object.

(5) In order to provide for the mapping of the normal blind spot, and for the quick determination and mapping of central and paracentral scotomata, a tangent screen is added subtending a visual angle of 60 or nore degrees. This screen can be quickly and conveniently attached to the stimulus carriage and moved into position.

With the controls provided, a careful worker can reproduce the limits of the color fields within 1 or 2 degrees.

Discussion. Dr. L. C. Peter said it was essential to be able to take fields which can be reproduced. His preference for the tangent screen in perimetric studies has been due to the fact that peripheral field records, altho not nearly so important as central studies, have been entirely unsatisfactory because of imperfect instruments. The sources of error in perimetric work from the clinical standpoint are illumination, variability of the intensity of the color stimulus, fixation, preexposure, and surrounding field. The perimeter exhibited seems to be as nearly accurate as its practical application in our routine office work demands, without complicating field taking too much by technicalities which will tend to minimize its use. He hoped that some means could be found to have one firm control the manufacture of the instrument, in order that uniformity in the intensity of the color stimuli and the grays necessary for preexposure and surrounding field may be preserved. Without careful attention to this important feature of the perimeter, much of its value will be missed. While Dr. Ferree did not discuss the size of the test object, he believed it should be designated in degrees or fractions of degrees rather than in millimeters.

Dr. Ziegler stated that he had found that electric illumination would yield results differing from daylight and inferior to it. In the first place, the color values are wrong; secondly, the color reactions differ from accepted standards, and thirdly, the fields are too large as compared with daylight findings. These deductions were based on the use of an electric perimeter with transilluminated colors.

\section{The Variable Factors Which Influence the Determination of the Color Fields.}

Drs. C. S. Ferree and G. RaNi, of Bryn Mawr College, said that the variable factors which influence the apparent limits of color sensitivity are the wave length and purity of the stimulus, the intensity of the stimulus and the visual angle, the length of exposure of the eye, the method of exposure (moving or stationary stimulus), accuracy and steadiness of fixation, the intensity of the general illumination of the retina and its state of adaptation, breadth of pupil, and the brightness of the preexposure and of the background or surrounding field. The most important of these from the standpoint of the work of the office and clinic are the intensity of the stimulus, the brightness of the preexposure and 
surrounding field, the intensity of the general illumination and the accuracy and steadiness of fixation.

Intensity of Stimulus.-By a sufficiently wide variation in this factor alone the fields of color sensitivity may be made to have almost any breadth within the field of vision, to differ radically in shape and even to change or reverse their order of ranking with regard to breadth. For example, with very high intensities the limits of red, yellow and blue are coincident with the limits of white light vision. Green cannot be made to have so wide an extent. With stimuli of medium intensity and of the relative energies found in the prismatic spectrum of a Nernst filament, the limits are concentric and in the order from widest to narrowest of red, yellow, blue and green. With stimuli of medium intensity of equal energy the limits of red, yellow and blue interlace or crisscross. The limits for green again are narrower. The limits for pigment stimuli may be either interlacing or concentric in the order of red, blue and green or blue, red and green depending upon the intensity of light falling on the perimeter arm. It seems only fair $t_{0}$ conclude, therefore, that the conventional clinic rating of the limits from widest to narrowest in the order of blue, red and green is a function of the relative and absolute intensities of the stimuli employed as well as of the actual distribution of sensitivities. Without great precision in the control of intensity, it is obvious that reproducibility of result cannot be obtained and little significance can be attached to extent or shape of field, to order of ranking as to breadth of field, or to any vatiations from time to time or from person to person in these important features.

BRIGITTNESS OF PREEXPOSURE AND SURROUNDING FIELD.-The brightness of the surface to which the eye is preexposed may change the apparent limits in certain meridians as much as 17 degrees. A preexposure lighter than the color gives a dark after image; a preexposure darker than the color, a light after image. These after images change profoundly the saturation of the color, also its hue. The brightness of the surrounding field, thru physiologic induction, exercises a similar effect but not so great in amount. The variable effects both of the preexposure and of the surrounding field are strongly influenced by changes in the intensity of the illumination. When the results are obtained under such ranges of change of illumination as may occur during the course of a given day or from day to day, the variability of effect is greatly increased, reaching in some meridians as much as $28-30$ degrees. Further important effects of surrounding field as influenced by change of illumination are the changes in hue which the color undergoes in passing towards the periphery of the retina.

Cinarles R. Hefi, M. D. Clerk.

\section{COLORADO OPHTHALMOLOGI- CAL SOCIETY.}

$$
\text { May 21, } 1921 .
$$

\section{DR. J. J. Pattee presiding.}

\section{Tuberculous Iridocyclitis.}

F. E. Wallace, Pueblo, again showed a negro boy, aged sixteen years, who had been presented to the society in October, 1920, on account of a severe chronic irido-cyclitis of both eyes, especially the right, and characterized in the right eye by the presence of a number of one $\mathrm{mm}$. deposits on the posterior surface of the cornea, arranged as a triangle with its apex downward. In the right eye there had also been a number of deposits at the angle of the anterior chamber, some of them two $\mathrm{mm}$. in diameter, as well ar deposits on the surface of the iris.

The patient had been very irregula. in attendance and in his use of medicine, and had disappeared entirely for nearly six months. The right cornea was now somewhat clearer, and the deposits on Descemet's membrane and on the iris had disappeared; those on Descemet's membrane being replaced by a thin plastic exudate plastered on the membrane at about six o'clock. This exudate was arranged in a triangular 